\title{
Methylation-associated inactivation of LATS1 and its effect on demethylation or overexpression on YAP and cell biological function in human renal cell carcinoma
}

\author{
KE-HONG CHEN ${ }^{1}$, JIANG HE ${ }^{2}$, DE-LIN WANG ${ }^{1}$, JIAN-JIA CAO ${ }^{1}$, MEI-CAI LI ${ }^{1}$, \\ XIU-MIN ZHAO ${ }^{1}$, XIA SHENG ${ }^{1}$, WEN-BIN LI ${ }^{1}$ and WU-JIANG LIU ${ }^{3}$ \\ ${ }^{1}$ Department of Urology, The First Affiliated Hospital of Chongqing Medical University, Chongqing; \\ ${ }^{2}$ Gastroenterology and Neurology Center, University-Town Hospital of Chongqing Medical University, \\ Chongqing; ${ }^{3}$ Institute of Urology, Peking University First Hospital, Beijing, P.R. China
}

Received July 22, 2014; Accepted September 16, 2014

DOI: 10.3892/ijo.2014.2687

\begin{abstract}
Large tumor suppressor 1 (LATS1) gene is one of the key factors in Hippo signaling pathway. Inactivation of LATS1 by promoter methylation was found in colorectal cancer (CRC), head and neck squamous cell carcinoma (HNSCC), astrocytoma, breast cancer and it was proved to be a tumor suppressor. However, its role is unclear in renal cell carcinoma (RCC). In this study, the expression of LATS1 was determined by reverse transcription polymerase chain reaction (RT-PCR) and immunohistochemistry in 30 pairs of RCC tissues and matched normal kidney tissues and RCC cells. We found that the expression of LATS1 was markedly reduced in RCC tissues and cells, in the RCC tissue in $46.7 \%$ (14/30), while in the normal kidney tissues in $76.7 \%$ (23/30), and was associated with pathological grade and clinical stage of RCC. We detected methylation status of LATS1 by bisulfite sequence-PCR (BSP) in renal cancer cell line 786-O which lowers expression of LATS1, and we found it hypermethylated (in 97.5\%). In addition, pharmacological demethylation using 5-Aza-2'-deoxycytidine (5-Aza) restored the expression of LATS1 mRNA and protein in 786-O cells, both LATS1 demethylation and overexpression of LATS1 downregulated the expression of Yes-associated protein (YAP), inhibited cell proliferation, induced cell apoptosis and cell cycle G1 arrest in 786-O cells. Thus, this report for the first time demonstrates the inactivation of LATS1 by promoter methylation and it is a tumor suppressor in kidney cancer. LATS1 may serve as a
\end{abstract}

Correspondence to: Professor De-Lin Wang, Department of Urology, The First Affiliated Hospital of Chongqing Medical University, 1 Youyi Road, Yuzhong District, Chongqing 400016, P.R. China

E-mail: dlwangws@sina.com

Key words: LATS1, renal cell carcinoma, methylation, demethylation, Hippo, YAP biomarker for possible early diagnosis and as a potential therapeutic target for human RCC.

\section{Introduction}

Renal cell carcinoma (RCC) has the highest fatality rate in urinary tract malignant tumors and accounts for 2-3\% of all cancers worldwide, $>102,000$ RCC patients die annually of the malignancy, the main type of RCC is classified as clear cell renal cell carcinoma (ccRCC) $(1,2)$. Due to RCC resistance to chemotherapy and radiotherapy, the mortality of patients with advanced RCC is very high. The localized RCC mainly relied on the surgical treatment, while therapy options of advanced RCC are very limited (3). Recent reports pointed out that the molecular target drugs (e.g., sunitinib, and sorafenib) were more effective in metastatic RCC (mRCC) and improved the survival rate of patients with $\mathrm{mRCC}$, but the long-term effect of these drugs were limited to $\operatorname{mRCC}(4,5)$. The molecular characteristics of RCC are complex and its mechanism needs clarification (6).

Epigenetic alteration, especially aberrant hypermethylation of the promoter region within a $\mathrm{CpG}$ island is involved in silencing of transcription of classical tumor suppressor genes (TSGs) in cancers and it was considered to be one of the earliest and most frequent alterations in cancer (7), which had two main specific mechanisms: one is that DNA methylation may inhibit gene expression directly by blocking binding to DNA of factors required for optimal transcription (8). The other is that methylation affects gene expression directly by interfering with transcription factor binding, and/or indirectly by recruiting histone deacetylases through methyl-DNA-binding proteins (9), however, the gene methylation is reversible, unlike mutation or loss of heterozigosity (LOH). Therefore, we should search for novel TSGs by way of promoter CpG methylation so as to reveal the epigenetic mechanism of carcinogenesis and also identify potential tumor biomarkers for early detection of RCC (10). At present, many candidate TSGs silenced by DNA methylation modification also have been reported in RCC, such as RASSF1 (11), DLC1 (12), and LRRC3B (13). 
The large tumor suppressor 1 (LATS1) gene has been identified as a TSG in Drosophila and encodes a putative serine/threonine kinase at the earliest time, which is a member of the nuclear Dbf2-related (NDR) family (14). LATS1 gene is located at chromosome 6q25.1 and its open reading frame is 3,393 bp encoding a 1130 -amino acid polypeptide with molecular weight of $126.87 \mathrm{kDa}$, which is highly similar to LATS2 in structure and function (15). Recently LATS1 has been identified as a key factor of the Hippo signaling pathway that plays pivotal roles in various biological processes such as cell proliferation, genetic stability, cell migration, cell metastasis, tumorigenesis, organ size control, stem cell differentiation and renewal, drug resistance, spindle formation, actin polymerization of modulation (16-19). However, studies have shown that the expression of LATS1 was reduced or in deficient in a wide variety of tumors, including gliomas (15), cervical cancer (20), gastric cancer (21), skin cancer (22), and metastatic prostate cancer (23). LATS1 knockout mice spontaneously developed non-metastatic soft tissue sarcomas and metastatic ovarian stromal cell tumors (24). Therefore, LATS1 has been considered as a TSG, but its role in human cancer is unclear (25). Recently, LATS1 downregulated by promoter hypermethylation has been reported in various human tumors including colorectal cancer (CRC) (26), head and neck squamous cell carcinoma (HNSCC) (27), soft tissue sarcoma (28), astrocytoma (29), and breast cancer (30), which indicated that LATS1 promoter hypermethylation was related to tumorigenesis. Thus, LATS1 may be a potential target gene for cancer gene therapy. However, whether LATS1 is subjected to epigenetic silencing and its function in RCC is unclear.

In this study, we hypothesized that LATS1 is epigenetically downregulated and functions as a TSG in RCC. To test this point of view, we first dectected the expression of LATS1 in RCC tissues by immunohistochemistry and reverse transcription polymerase chain reaction (RT-PCR) and analyzed its relationship with the clinicopathologic characteristics of RCC, then selected 786-O cells with low expression of LATS1 mRNA, which promoter methylation was examined by bisulfite sequence-PCR (BSP), we subsequently investigated the effects of LATS1 gene demethylation and overexpression on the biological function and Yes-associated protein (YAP) in human RCC 786-O cells. The ultimate goal of this report is to determine whether LATSI can be used as a potential target gene for RCC diagnosis and therapy.

\section{Materials and methods}

Patients and tissue specimens. The study was approved by the local Ethics Committees and informed written consent was obtained. Permission for the use of tissue, it was obtained from the First Affiliated Hospital of Chongqing Medical University from March to December 2012. Eligible patients were diagnosed to have clear cell carcinoma of the kidney by pathological methods. The total number of patients was 30,15 male and 15 female, aged 36-77 with an average age of 63 . We chose the RCC tissues and matched normal kidney tissues ( $4 \mathrm{~cm}$ away from the cancer tissues) undergone radical nephrectomy. Each specimen was divided into two equal parts, one was drawn into a Haoe frozen tube after being treated by diethylpyrocarbonate (DEPC) water, liquid nitrogen frozen condensate, cryopreserved at $-80^{\circ} \mathrm{C}$ for RT-PCR experiments; the other was placed in $10 \%$ formalin solution for the immunohistochemistry experiment.

Immunohistochemisty. The tissues were fixed with $10 \%$ formaldehyde (ZSGB-BIO, Beijing, China) embedded in paraffin, sectioned into $4 \mu \mathrm{m}$ thick slices and used for staining. In brief, anti-LATS1 (1:100) (Santa Cruz Biotechnology, Inc., Dallas, TX, USA) antibodies were applied to the paraffin section, after deparaffinization, antigen reconditioning and serum (Gibco-BRL, Carlsbad, CA, USA) was blocked. Then incubated in $37^{\circ} \mathrm{C}$ water bath for $2 \mathrm{~h}$ and the secondary antibody and streptomycin antibiotics-peroxidase was applied on the section. Visualization was performed using DAB chromogen. Sections were restained with hematoxylin (Shanghai BlueGene Biotech Co., Ltd., Shanghai, China), dehydrated, and mounted in neutral gum, and analyzed using a bright field microscope. The tissues were scored according to positive areas and staining intensity. The percentage of positive areas was graded as $0(\leq 5 \%), 1(6-25 \%), 2(26-50 \%), 3(\geq 51 \%)$, and the staining intensity was graded as $0-2$ (i.e., 0 , negative; 1 , weak; 2 , strong). The two grades were multiplied and tissues were assigned to one of three levels: 0 was negative, 1-4 was weak positive, 5-6 was strong positive.

Cell culture and 5-Aza. 786-O and HEK-293 were purchased from American Type Culture Collection (ATCC) (Rockville, MD, USA), 786-O cells were maintained in RPMI-1640 containing 10\% Gibco fetal bovine serum (FBS) (Gibco-BRL) with $1 \%$ antibiotics. HEK-293 cells were grown in DMEM/HG supplemented with FBS and antibiotics. All cells were maintained at $37^{\circ} \mathrm{C}$ in a humidified incubator with $5 \%$ $\mathrm{CO}_{2}$. Cells were treated with 5-Aza-2'-deoxycytidine (5-Aza) (Sigma-Aldrich, St. Louis, MO, USA) $1 \mu \mathrm{M}$ for 4 days. Culture medium and 5-Aza were replaced daily.

RNA isolation and RT-PCR. Total RNA was isolated from RCC cells or tissues using RNAiso Plus (Takara Bio, Inc., Osaka, Japan). The total RNA quality was detected by UV spectrophotometer (Bio-Rad, Hercules, CA, USA), $1 \mu \mathrm{g}$ RNA was reverse to synthesis the single-stranded cDNA using PrimeScript RT reagent kit and then amplified with Premix Taq Q3 2.0 kit (both from Takara Bio, Inc.) according to the manufacturer's instructions. The primers used are as follows. LATS1 forward, 5'-CCACCCTACCCAAAACATCTG-3' and reverse, 5'-CGC TGCTGATGAGATTTGAGTAC-3'; YAP forward, 5'-TGA ACAAACGTCCAGCAAGATAC-3' and reverse, 5'-CAGCCC CCAAAATGAACAGTAG-3'. GAPDH was used as internal control. GAPDH forward, 5'-ACCACCATGGAGAAGGC TGG-3' and reverse, 5'-CTCAGTGTAGCCCAGGATGC-3'. PCR conditions were as follows: $94^{\circ} \mathrm{C}$ for $5 \mathrm{~min}$, followed by 30 cycles of $94^{\circ} \mathrm{C}$ for $30 \mathrm{sec}, 56^{\circ} \mathrm{C}$ for $60 \mathrm{sec}$, and $72^{\circ} \mathrm{C}$ for $60 \mathrm{sec}$. The PCR products were run on a $2 \%$ agarose gel. The relative expressions of LATS1 and YAP were quantitatively measured using densitometry by Quantity One software (Bio-Rad).

Western blot analysis. Cells were scraped and lysates were prepared in $80 \mu \mathrm{l}$ of radioimmunoprecipitation assay (RIPA) lysis buffer containing $1 \%$ phenylmethanesulfonyl fluoride (PMSF), and protein concentration was determined by 


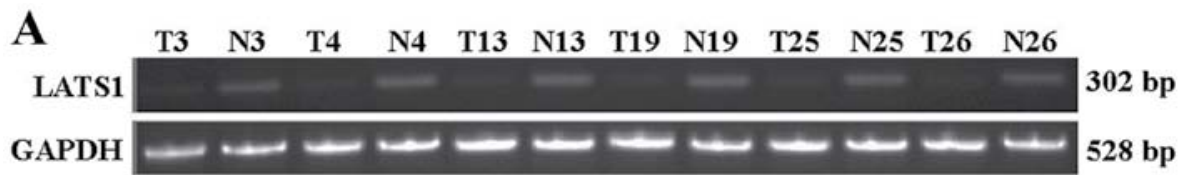

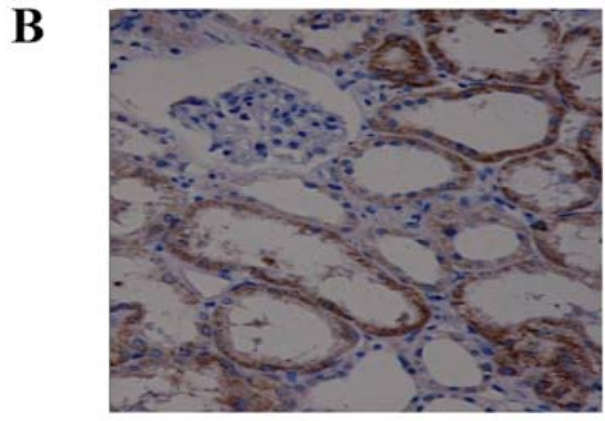

Normal renal tissues

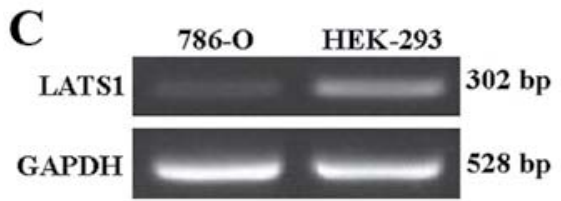

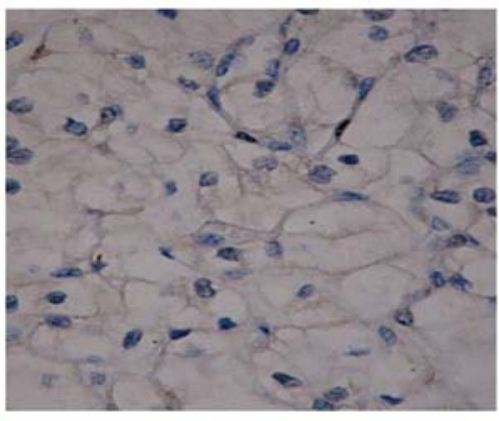

RCC tissues

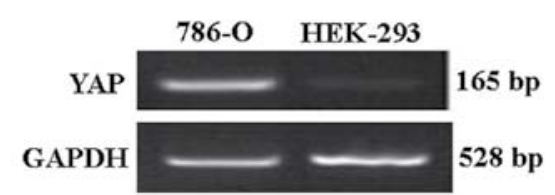

Figure 1. Expression of large tumor suppressor 1 (LATS1) in renal cell carcinoma (RCC) tissues and cells. (A) Expression of LATS1 mRNA in random RCC tissues and normal kidney tissues. T, RCC tissues; N, normal kidney tissues. (B) The expression of LATS1 protein in RCC tissues and normal kidney tissues by immunohistochemistry (x400). (C) Expression of LATS1 and Yes-associated protein (YAP) mRNA in 786-O and HEK-293 cells.

protein quantitated kit (all from Beyotime, Shanghai, China). After mixing with loading buffer and denaturalizing by boiling for $10 \mathrm{~min}, 50 \mu \mathrm{g}$ of protein was loaded and separated on $6 \%$ (for LATS1) or $10 \%$ (for YAP and $\beta$-actin) sodium dodecyl sulfate polyacrylamide gel electrophoresis (SDS-PAGE) at $80 \mathrm{~V}$. The proteins were transferred onto polyvinylidene difluoride (PVDF) membranes (Millipore, Billerica, MA, USA) at $250 \mathrm{~mA}$. The membranes were blocked by $5 \%$ non-fat dry milk in TBS containing $0.05 \%$ Tween-20 (TBST) for $2 \mathrm{~h}$ at room temperature, primary antibodies against LATS1 (Santa Cruz Biotechnology, Inc.) at 1:150, YAP (Santa Cruz Biotechnology, Inc., Santa Cruz, CA, USA) at 1:200, and $\beta$-actin (Beyotime) at 1:500 were applied overnight at $4^{\circ} \mathrm{C}$, membranes were washed with TBST, and then incubated with horseradish peroxidase-conjugated goat anti-rabbit secondary antibody (Bioworld Technology Co., Ltd., Jiangsu, China) for $1 \mathrm{~h}$ at $37^{\circ} \mathrm{C}$. The proteins of interest were visualized with the enhanced ECL detection system (Beyotime). The densitometry of band was quantified by Quantity One software (Bio-Rad).

BSP. Cells were collected and washed with PBS. BSPs were carried out as described previously (31), DNA was extracted from the cells using TIANamp Genomic DNA kit [Tiangen Biotech (Beijing) Co., Ltd., Beijing, China] according to the manufacturer's protocol, bisulfite-treated DNA was PCR amplified using primers BSP forward, 5'-AGAAGAAAGTTT TGGATTTATTAAAT-3' and reverse, 5'-CATTTATAAATT AACTTCTAAAATAC-3'. The PCR products were electrophoresed and purified using Spin-X tubes, and then cloned into the pUC-T vector (both from CWbiotech, Beijing, China), with 10 colonies randomly chosen and sequenced.
Lentiviral vectors and infection. To overexpress LATS1 in 786-O cells, LATS1-expressing lentiviruses were generated using the GV218 system (Shanghai GeneChem Co., Shanghai, China) containing the full-length coding region (from GAGGAT CCCCGGGTACCGGTCGCCACCATGAAGAGGAGTGAA AAGCCAGAAGG to TCACCATGGTGGCGACCGGAA CATATACTAGATCGCGATTTTTAATC) of LATS1. The recombinant virus was purified, and titrated by qPCR, and its infectivity was detected after transduction at increasing multiplicity of infection (MOI). The experiment was divided into three groups: control group, mock virus and lentiviral-LATS1.

Flow cytometry analysis (FCM). To evaluate the cell apoptosis and cell cycle, the cells were digested and adjusted in density of $1 \times 10^{6} / \mathrm{ml}$, washed two times with PBS and add $1 \mathrm{ml}$ PBS to beat it after centrifugal, cells were stained with Annexin V-FITC (Nanjing KeyGen Biotech Co., Ltd., Nanjing, China) and propidium iodide (PI) (BD Biosciences, Shanghai, China) for $30 \mathrm{~min}$ at room temperature and determined by FCM (Becton-Dickinson, Franklin Lakes, NJ, USA) to detect the cell apoptosis. According to the aforementioned method, the cells were centrifugated, fixed with $70 \%$ ethanol, and incubated for $10 \mathrm{~min}$ with $2 \mathrm{mg} / \mathrm{ml}$ RNase (Sigma-Aldrich), the cellular DNA was then stained with $50 \mathrm{ng} / \mathrm{ml}$ PI for $30 \mathrm{~min}$ at room temperature in darkness, and the cell cycle was analyzed by FCM.

Cell counting kit-8 (CCK-8). The cells were seeded in 96-well plates. After demethylation or infection, the cell proliferation was determined by using a CCK-8 (Nanjing KeyGen 
Biotech Co., Ltd.) following manufacturer's instructions. The optical density (OD) was measured with a microplate reader (SpectraMax M2; Molecular Devices, Sunnyvale, CA, USA) at $450 \mathrm{~nm}$ wavelength, then the cell proliferation inhibition rate (IR) was calculated.

Statistical analysis. Statistical analyses were performed with SPSS, version 19.0 (SPSS, Inc., Chicago, IL, USA). Data are shown as mean values \pm standard deviation (SD). Differences between the two independent groups were analyzed by the Student's t-test. The $\chi^{2}$ test was used to calculate differences in the patients' age, gender, tumor stage, clinical stage and pathological grade. $\mathrm{P}<0.05$ was considered significant.

\section{Results}

Expression of LATS1 in RCC tissues and cell lines. We examined the expression of LATS1 in 30 pairs of RCC tissues and matched normal kidney tissues by RT-PCR and immunohistochemistry. RT-PCR results (Fig. 1A) showed that the expression of LATS1 mRNA was significantly decreased $(\mathrm{P}<0.05)$ in RCC tissues, while the expression of LATS1 mRNA in normal kidney tissues was increased.

We examined the expression of LATS1 mRNA in 786-O and HEK-293 cell line by RT-PCR. As shown in Fig. 1C, compared with HEK-293, the expression of LATS1 mRNA was significantly decreased $(\mathrm{P}<0.05)$ in $786-\mathrm{O}$.

Immunohistochemistry revealed that the expression rate of LATS1 in the RCC tissue was $46.7 \%$ (14/30) and negative or weakly positive, while the expression rate of LATS1 in the normal kidney tissues was $76.7 \%$ (23/30) and strongly positive (Fig. 1B). LATS1 mainly accumulated in cytoplasm of kidney tubules and presented as brown-yellow or brown particles.

LATS1 expression is related with clinicopathologic characteristics of $R C C$. The relationships between LATS1 expression with the clinicopathologic characteristics in RCC was analyzed. We did not find a significant correlation of the expression of LATS1 with patients' gender, age, tumor size and renal vein metastasis in $\mathrm{RCC}(\mathrm{P}>0.05)$. However, we observed that the expression of LATS1 was related to the clinical stage and pathological grade in RCC (Table I).

Methylation status of the LATS1 promoter region. The LATS1 CpG island is located in chromosome 5' UTR of 6q24-25 (32). We selected 786-O with low expression of LATS1 mRNA, and the methylation status at eight $\mathrm{CpG}$ sites of the LATS1 $\mathrm{CpG}$ islands from -600 to $500 \mathrm{bp}$ was characterized by BSP. This analysis indicated that the $\mathrm{CpG}$ islands were densely methylated in 786-O cells. The methylation rate accounted for 97.5\% (Fig. 2).

Restoration of LATS1 expression and downregulation of YAP expression by treatment with 5-Aza demethylation. To test whether methylation directly induced LATS1 silencing, we demethylated the LATS1 gene in 786-O cells and HEK-293 cells with 5-Aza, an inhibitor of DNA methyltransferases (DNMTs). As shown in Fig. 3, after treating with $1 \mu \mathrm{M} 5$-Aza for $96 \mathrm{~h}$, the mRNA and protein of LATS1 were restored, while the mRNA
Table I. The correlation of LATS1 protein expression with clinicopathologic characteristics in $\mathrm{RCC}\left(\chi^{2}\right.$ test).

\begin{tabular}{|c|c|c|c|c|c|}
\hline \multirow[b]{2}{*}{ Group } & \multirow[b]{2}{*}{ No. } & \multicolumn{3}{|c|}{ LATS 1 expression } & \multirow[b]{2}{*}{$\mathrm{P}$} \\
\hline & & $(+)$ & $(-)$ & $\begin{array}{l}\text { Positive } \\
(\%)\end{array}$ & \\
\hline Gender & & & & & 0.464 \\
\hline Male & 15 & 6 & 9 & 40 & \\
\hline Female & 15 & 8 & 7 & 53.3 & \\
\hline Age (years) & & & & & 0.232 \\
\hline$<60$ & 18 & 10 & 8 & 55.6 & \\
\hline$\geq 60$ & 12 & 4 & 8 & 33.3 & \\
\hline $\begin{array}{l}\text { Renal vein } \\
\text { metastasis }\end{array}$ & & & & & 0.171 \\
\hline Yes & 2 & 0 & 2 & 0 & \\
\hline No & 28 & 14 & 14 & 50 & \\
\hline $\begin{array}{l}\text { Size of } \\
\mathrm{RCC}(\mathrm{cm})\end{array}$ & & & & & 0.295 \\
\hline$>5$ & 8 & 5 & 3 & 62.5 & \\
\hline$\leq 5$ & 22 & 9 & 13 & 40.9 & \\
\hline $\begin{array}{l}\text { Pathological } \\
\text { grading }\end{array}$ & & & & & 0.024 \\
\hline Well & 8 & 6 & 2 & 75 & \\
\hline Moderate & 13 & 7 & 6 & 53.8 & \\
\hline Poor & 9 & 1 & 8 & 11.1 & \\
\hline $\begin{array}{l}\text { Clinical } \\
\text { staging }\end{array}$ & & & & & 0.024 \\
\hline I-II & 17 & 11 & 6 & 64.7 & \\
\hline III-IV & 13 & 3 & 10 & 23.1 & \\
\hline
\end{tabular}

LATS1, large tumor suppressor 1; RCC, renal cell carcinoma.

and protein of YAP were downregulated in 786-O cells, but, there was no obvious change in HEK-293 cells. These results indicate that methylation directly mediates the transcriptional silencing of LATS1 in RCC.

Biological function is affected by LATS1 demethylation with 5-Aza. To investigate the effects of LATS1 gene demethylation on the biological function in human RCC cell line 786-O. According to the above, in the treatment of cells with $1 \mu \mathrm{M}$ 5-Aza for $96 \mathrm{~h}$, the FCM revealed that the apoptosis rate of $786-\mathrm{O}$ cells in experiment group $(27.73 \pm 2.85) \%$ was significantly higher than that of control group $(7.54 \pm 1.71) \%(\mathrm{P}<0.05)$, while the apoptosis rate of HEK-293 had no obvious difference in the experiment group $(16.16 \pm 0.94) \%$ from its control $(15.77 \pm 0.98) \%(\mathrm{P}>0.05)$. This result showed that demethylation of LATS1 induced apoptosis of 786-O cells (Fig. 4A).

In order to ascertain the cell cycle, the FCM showed that G1 stage $(82.12 \pm 3.01) \%$ of $786-\mathrm{O}$ cells in experiment group was significantly higher than that of control group $(57.43 \pm 1.13) \%(\mathrm{P}<0.05)$, while G1 stage $(61.14 \pm 1.05) \%$ of HEK-293 cells in experiment group had no obvious difference from its control group $(60.35 \pm 0.94) \%$ ( $\mathrm{P}>0.05)$. These results 


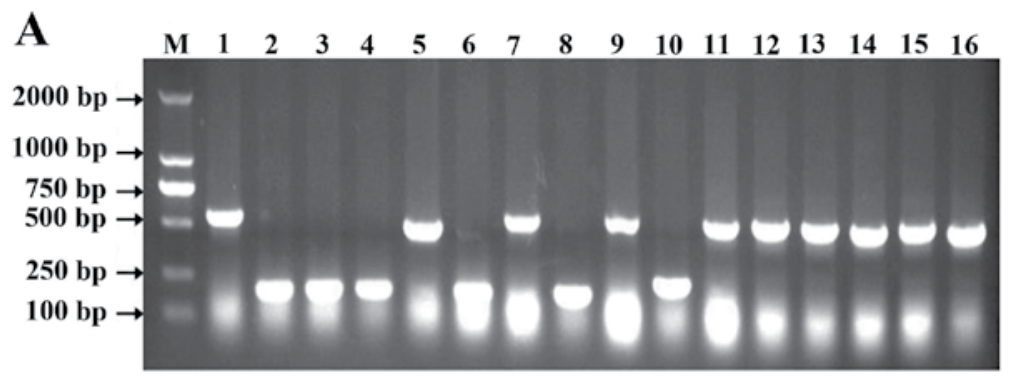

B

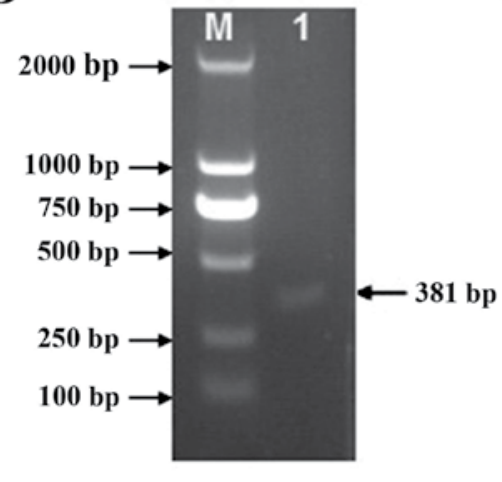

C

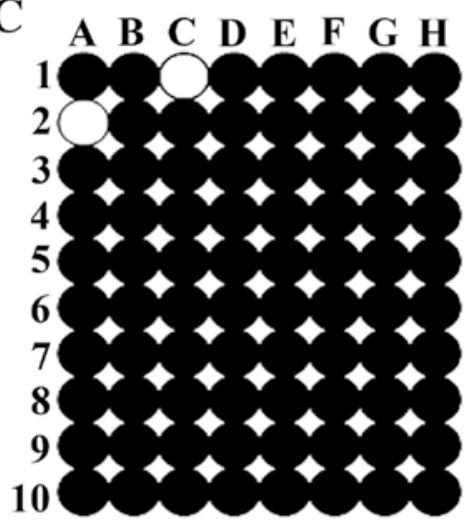

D

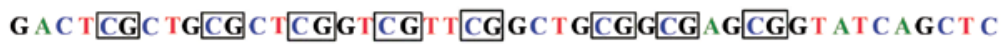

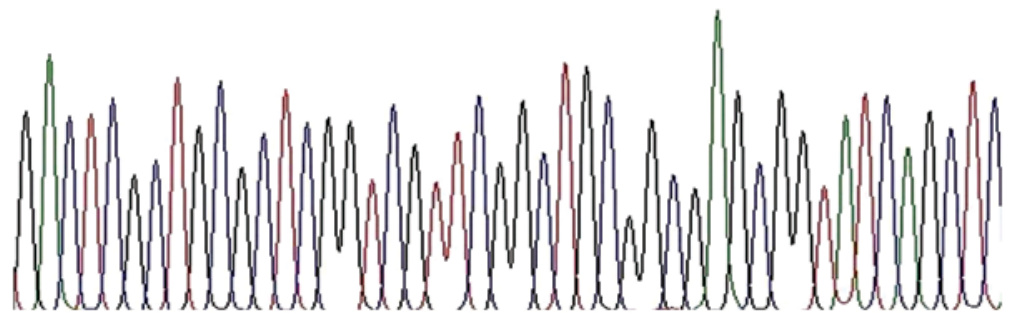

Figure 2. The large tumor suppressor 1 (LATS1) promoter methylation status was detected by bisulfite sequence-PCR (BSP). (A) Monoclonal bacterial colony was detected by PCR. M, Marker; 1 to 16 represent 16 clones, positive clones were located in 500 bp. (B) Use BSP primer to amplify LATS1. M, Marker; Lane 1, 786-O cell line. (C) The 1-10 represent 10 positive clones, A-H represent eight CpG islands, each row of circles represents an individual CpG site in LATS1 by BSP; filled circle, methylated; empty circle, unmethylated. (D) Representative sequencing data of LATS1 CpG methylation. Boxes in the figure represent LATS1 promoter regions from -600 to 500 bp in eight CpG islands.

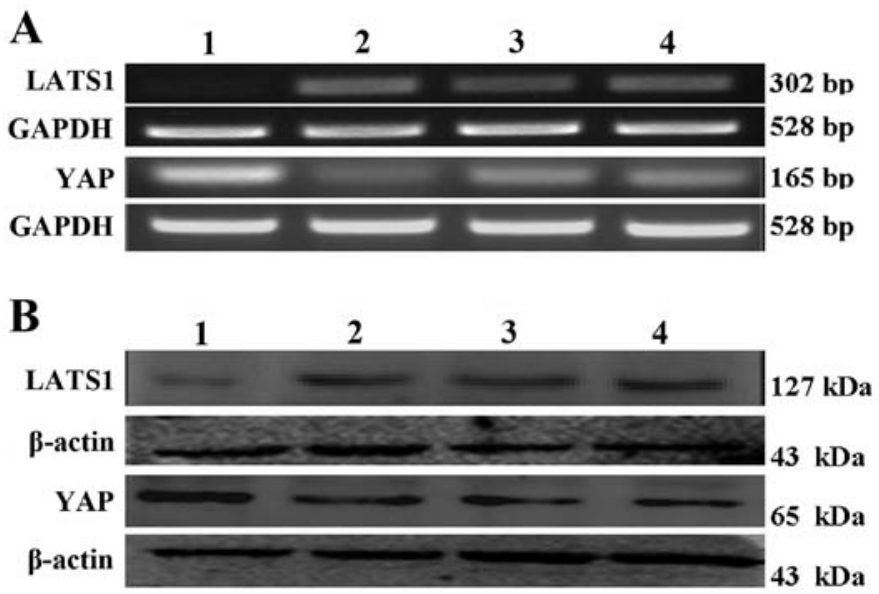

Figure 3. The expression of large tumor suppressor 1 (LATS1) and Yes-associated protein (YAP) after pharmacological demethylation using 5-Aza-2'-deoxycytidine (5-Aza) for each group. (A) The expression of LATS1 and YAP mRNA were detected by reverse transcription polymerase chain reaction (RT-PCR). (B) The expression of LATS1 and YAP protein were detected by western blot analysis. Lane 1, 786-O cells in control group; lane 2, 786-O cells treated with $1 \mu \mathrm{M}$ 5-Aza group; lane 3, HEK-293 cells in control group; lane 4, HEK-293 cells treated with $1 \mu \mathrm{M} 5$-Aza group. 

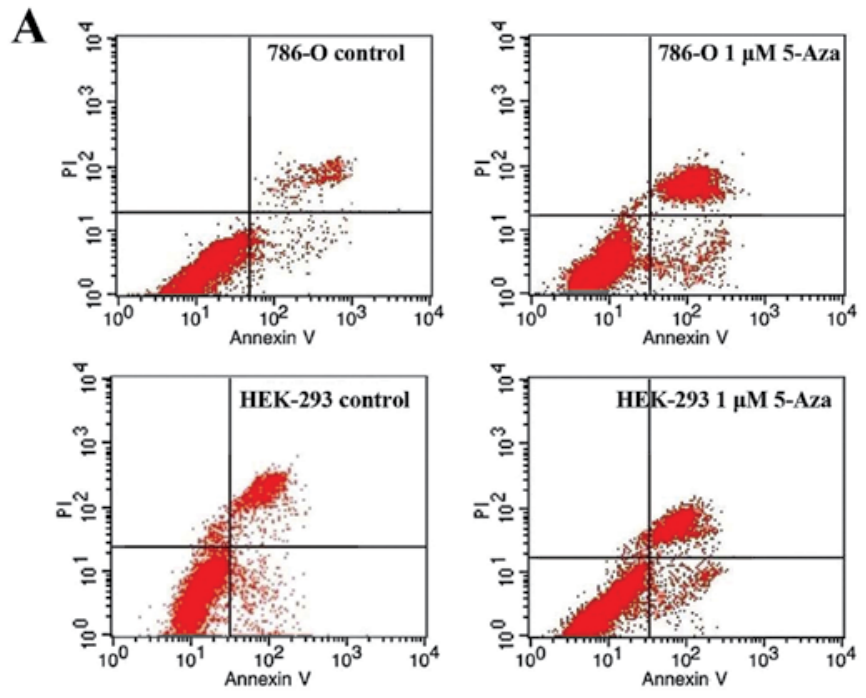

B
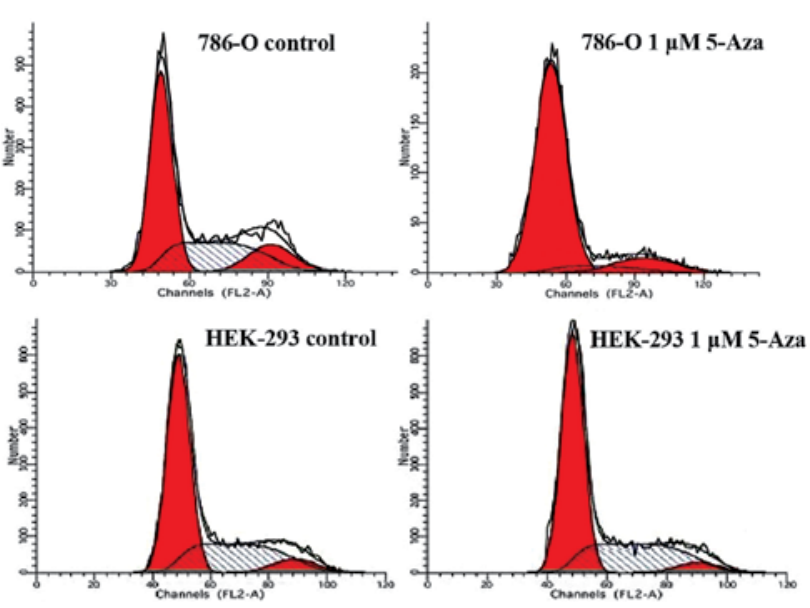

C

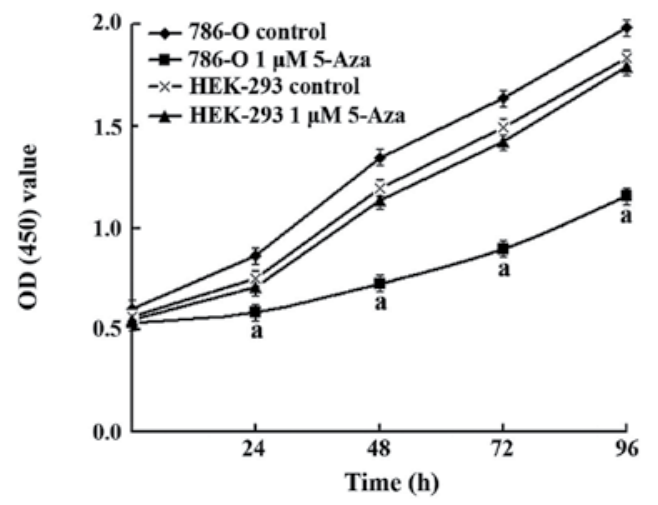

Figure 4. The biological function was detected by flow cytometry analysis (FCM) and cell counting kit-8 (CCK-8) after pharmacological demethylation using 5-Aza-2'-deoxycytidine (5-Aza) to deal with each group. (A) The apoptosis was detected by FCM. (B) The cell cycle was also detected by FCM. (C) The cell proliferation was detected by CCK-8. Cell growth curves were delineated and inhibition rate (IR) of cell proliferation was calculated by measuring absorbance at $450 \mathrm{~nm},{ }^{\text {a }} \mathrm{P}<0.05$.

indicate that demethylation of LATS1 induced 786-O cell cycle to arrest in G1 stage (Fig. 4B).

To investigate cell proliferation, CCK- 8 showed that the OD value $(1.16 \pm 0.01)$ of 786 - $O$ cells in the experimental group was obviously lower than that of the control group $(1.98 \pm 0.01)(\mathrm{P}<0.05)$ after treatment with $1 \mu \mathrm{M} 5$-Aza for $96 \mathrm{~h}$. The cell proliferation IR was 32, 46, 45, and 41\%, respectively, after 24, 48, 72, and $96 \mathrm{~h}$ of LATS1 demethylation, while the HEK-293 cells were not obviously inhibited. This result indicated that cell proliferation was markedly inhibited by LATS1 demethylation with 5-Aza in 786-O cells (Fig. 4C).

Lentiviral vectors mediated LATS1 overexpression and downregulated YAP. To test the effect of LATS1 on YAP in RCC cells, 786-O cells were infected with lentiviral-LATS1 (Shanghai GeneChem Co.) at MOI 60 for each viral vector after $96 \mathrm{~h}$. Proportion of infected 786-O cells was detected by FCM. Results showed that the transfection efficiency in control group, mock virus group and lentiviral-LATS1 group was 0.06, 95.96 and $81.69 \%$ (Fig. 5A), respectively. Expression of green fluorescent protein (GFP) after transfection was detected by fluorescence microscopy (Fig. 5B). The expression of LATS1 and YAP was detected at both mRNA and protein levels by RT-PCR and western blot analysis (Fig. 6). Compared with control group and mock virus group, the data show that the expression of LATS1 mRNA and protein in lentiviral-LATS1 group was dramatically increased $(\mathrm{P}<0.05)$, but the expression of YAP mRNA and protein was clearly decreased $(\mathrm{P}<0.05)$.

Effect of LATS1 overexpression on biological function. To test the functions of LATS1 in RCC cell 786-O, apoptosis in 786-O cells transduced by lentiviral-LATS1 after $96 \mathrm{~h}$ was determined by FCM (Fig. 7A), the percentage of apoptotic cells in the groups was: control group $(8.40 \pm 1.11) \%$, mock virus $(8.12 \pm 1.01) \%$, lentiviral-LATS1 $(22.76 \pm 1.09) \%$. Based on our data, it suggested that 786-O cells transduced by lentiviral-LATS1 induced cell apoptosis.

To investigate the effect of LATS1-mediation on the RCC cell cycle, the transfected 786-O cells were assayed by FCM. The results showed increasing numbers of cells arrested in G1 stage. The frequency of cells in G1 was $(58.20 \pm 1.27) \%$ in the control group, $(58.12 \pm 1.06) \%$ in mock virus group, and $(79.06 \pm 1.43) \%$ in the lentiviral-LATS1 group. These results suggested LATS1 caused G1 stage arrest $(\mathrm{P}<0.05)$ (Fig. 7B).

We used the CCK- 8 assay to determine the cell proliferation of 786-O cells which were transduced by lentiviral-LATS 1 . During the first 3 days, we found the OD value had no significant difference $(\mathrm{P}>0.05)$ in the three groups, but starting from the fourth day, the lentiviral-LATS1 group $(1.512 \pm 0.019)$ was strikingly lower than the control group $(1.808 \pm 0.02)(\mathrm{P}<0.05)$ and mock virus group $(1.763 \pm 0.014)(\mathrm{P}<0.05)$, and the cell proliferation IR was $4.71,5.43,3.70,16.37$, and $22.85 \%$, respectively, after transfecting cell for $24,48,72,96$, and $120 \mathrm{~h}$ by lentiviral-LATS1, but the mock virus group and control group were not obviously inhibited. The results presented in Fig. 7C show that cell proliferation was markedly inhibited by the LATS1 gene.

\section{Discussion}

The Hippo signaling pathway has been shown to be involved in tumorigenesis, and the core components of this pathway include MST1/2, WW45, LATS1/2, MOB1, and YAP, and they interact with each other (33). In mammals, when the pathway is activated by cell-cell contact or high cell density (34), a core kinase cascade causes a series of reactions (25), MST1/2 kinase 

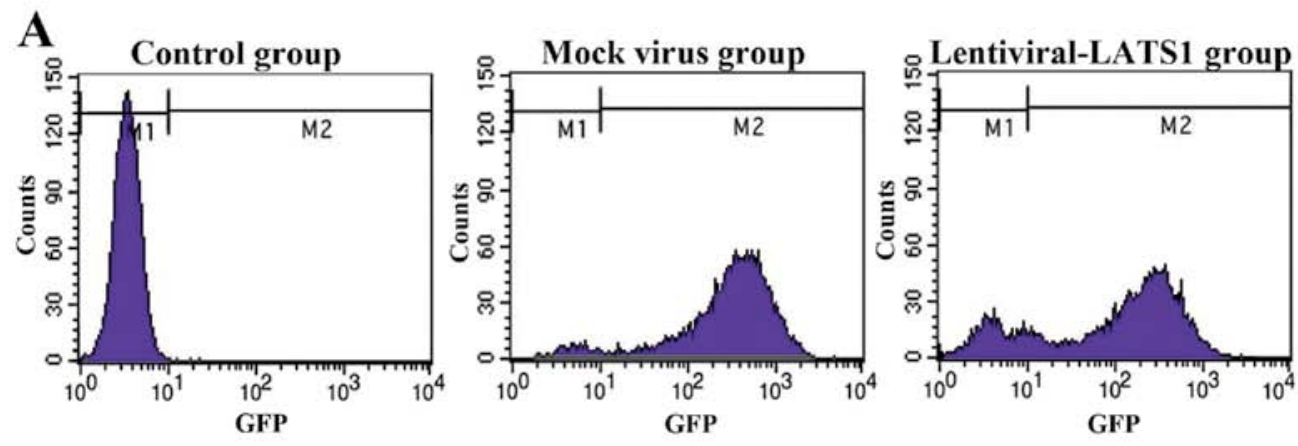

B
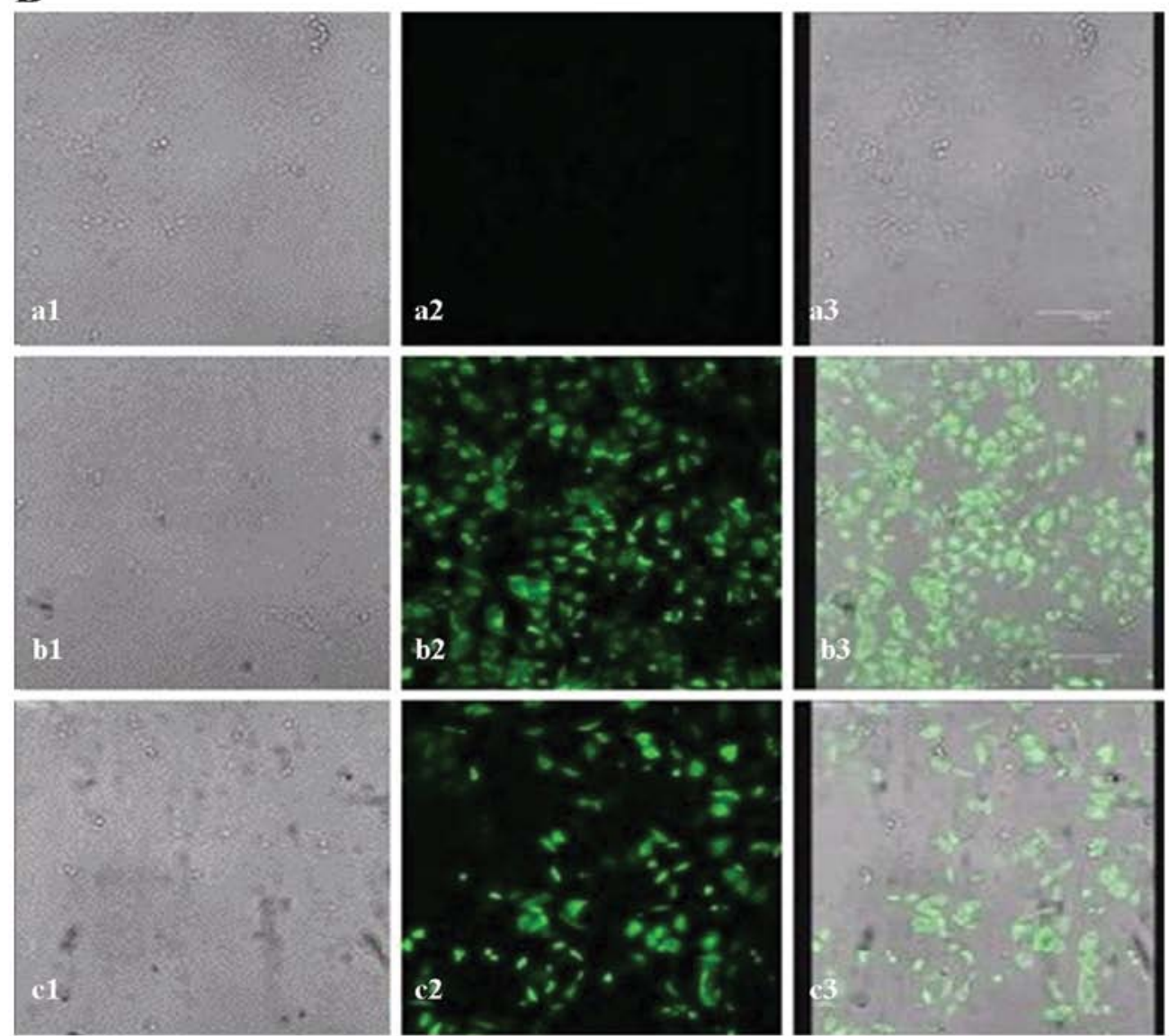

Figure 5. Expression of green fluorescent protein (GFP) in 786-O cells. (A) The transfection efficiency was assayed by flow cytometry analysis (FCM). M1, untransfected 786-O cells; M2, transfected 786-O cells. Control group represents untransfected 786-O cells; Mock virus group represents the empty vector transfected 786-O cells; Transfection efficiency was 95.96\% (M2); Lentiviral-large tumor suppressor 1 (LATS1) group represents lentiviral-LATS1 transfected 786-O cells. Transfection efficiency was 81.69\% (M2). (B) Expression of GFP after transfection was detected by fluorescence microscopy. a1, untransfected 786-O cells under optical microscope; a2, untransfected 786-O cells under fluorescence microscope; a3, the overlap figure of a1 and a2; b1, the empty vector transfected 786-O cells under optical microscope; b2, the empty vector transfected 786-O cells under fluorescence microscope; b3, the overlap figure of b1 and b2; c1, 786-O cells transfected with lentiviral-LATS1 under optical microscope; c2, 786-O cells transfected with lentiviral-LATS1 under fluorescence microscope; $\mathrm{c} 3$, the overlap figure of $\mathrm{c} 1$ and $\mathrm{c} 2$.
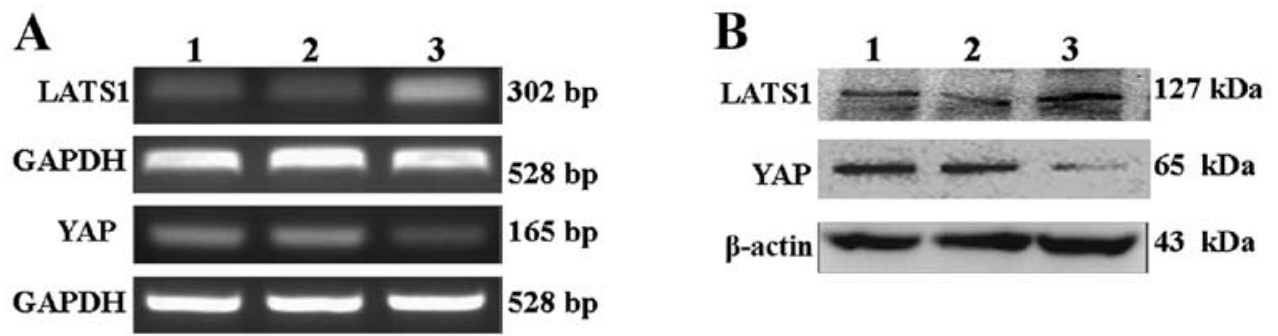

Figure 6. The expression of large tumor suppressor 1 (LATS1) and Yes-associated protein (YAP) in 786-O cells transfected with lentiviral-LATS1. (A) The expression of LATS1 and YAP mRNA in 786-O cells was detected by reverse transcription polymerase chain reaction (RT-PCR). (B) The expression of LATS1 and YAP protein in 786-O cells was detected by western blot analysis. Lane 1, control group; lane 2, mock virus group; lane 3, lentiviral-LATS1 group. 

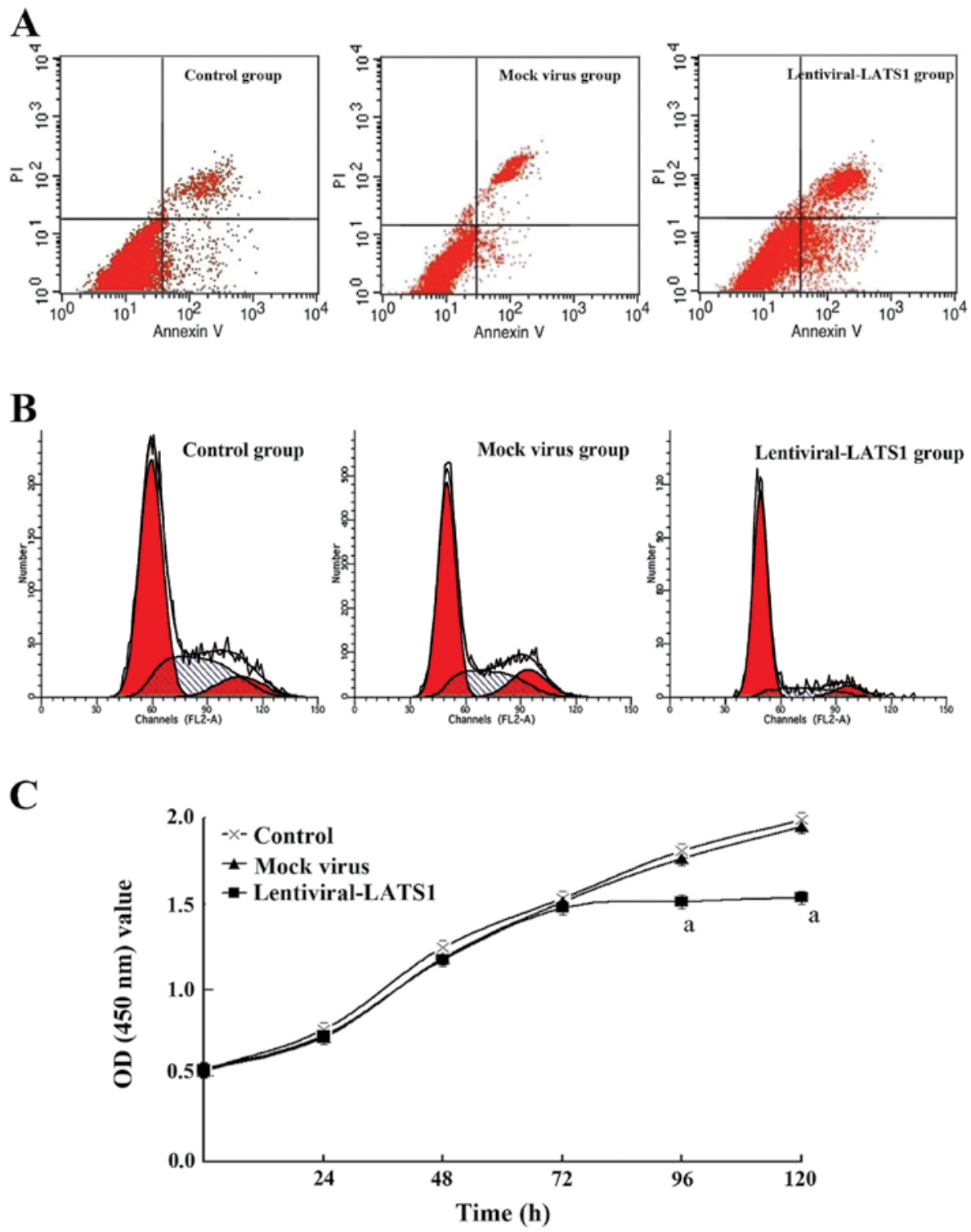

Figure 7. The biological function was evaluated by flow cytometry analysis (FCM) and cell counting kit-8 (CCK-8) after transfection with lentiviral-large tumor suppressor 1 (LATS1). (A) Effects of lentiviral-LATS1 on apoptosis in 786-O cells by FCM. (B) Effects of lentiviral-LATS1 on the cell cycle in 786-O cells by FCM. (C) The cell proliferation was detected by CCK-8. Cell growth curves were delineated and the inhibition rate of cell proliferation was calculated by measuring absorbance at $450 \mathrm{~nm},{ }^{a} \mathrm{P}<0.05$.

interacts with and phosphorylates WW45, an adaptor protein. Together, this complex phosphorylates and activates LATS1/2, which, together with its co-factor MOB1, phosphorylates YAP. Once phosphorylated, YAP is sequestered or degraded in the cytoplasm, which inhibits the expression of proliferation related genes cyclin DI and cyclin $E$ (35). If any one of the core components mentioned above changes, it will lead to the unlimited growth in tissues and organs, and eventually trigger tumors. Recent research shows, that Hippo signaling pathway, in addition to the core components found above, can interact with KIBRA (36) and FRMD6 (37). Although these factors may belong to the Hippo signaling pathway, how LATS1 is negatively regulated is largely unknown. Research over the past decades has revealed that LATS1 is a central part of a complex signal transduction cascade in multicellular eukaryotes (38) and a regulator in cellular homeostasis (39). Absence of LATS1 would lead to the formation of a variety of cancers, including gliomas (15), cervical cancer (20), gastric cancer (21), skin cancer (22), metastatic prostate cancer (23), ovarian stromal cell tumors (24). However, the expression of LATS1 in RCC is unclear.

In this study, we first demonstrated that LATS1 was silenced in RCC tissues and found LATS1 mainly accumulated in cytoplasm of kidney tubules by immunohistochemistry. This suggested RCC is likely to begin in the renal tubules. Furthermore, the expression of LATS1 protein was related with clinicopathologic characteristics of RCC, and the expression of LATS1 was related to the tumor clinical stage and pathological grade. We subsequently measured the expression of LATS1 mRNA in human RCC tissues and matched normal kidney tissues, and found that the expression of LATS1 mRNA was significantly decreased in RCC tissues. We further validated the downregulation of LATS1 mRNA in RCC cells by RT-PCR. The results suggested that the decreased expression of LATS1 contributes to RCC progression and may play a role of TSG in tumorigenesis of RCC. 
Though studies have shown that LATS1 inactivation was caused by other mechanisms, including LATS1 regulated by ubiquitination regulatory factors (40), integrin-linked kinase (ILK) (41), protease-activated receptors (PARs) (42), G protein-coupled receptor (GPCR) signaling pathway (43), LATS1 was supposed to be inactivated in three major mechanisms: $\mathrm{LOH}$, gene mutations, and hypermethylation of its promoter region (32). Despite the relatively high frequency of $\mathrm{LOH}$ at the locus containing LATS1 reported in breast cancers $(44,45)$, only one specimen with reduced LATS1 expression was demonstrated with an allelic LOH and somatic mutation of LATS1 was not detected in 25 breast cancers by RT-PCR-SSCP (46). Takahashi et al (30) reported that methylation frequency of LATS1 was $56.7 \%$ in breast cancer, which indicated that hypermethylation of the promoter regions of LATS1 is likely to play an important role in the downregulation of mRNA levels in breast cancers. These results showed LATS1 was unlikely to be inactivated in such a classic way as a combination of $\mathrm{LOH}$ and somatic mutation, but was more likely to be induced by hypermethylation. Therefore, we selected the 786-O cells which decreased LATS1, and analyzed promoter methylation of LATS1 at eight CpG sites from -600 to 500 bp by BSP. We found its promoter was densely methylated, the methylation rate accounted for $97.5 \%$. Other similar results were also reported, Wierzbicki et al (26) found that the promoter regions of LATS1 was hypermethylated as high as $57 \%$ in 44 CRCs, and they concluded that decreased expression of LATS1 in CRC was associated with promoter hypermethylation, but not microsatellite instability (MSI) status. Steinmann et al (27) analyzed the promoter methylation of LATS1 in 54 HNSCC specimens, and found that its hypermethylation accounted for $24 \%$, and that a trend of increased LATS1 methylation in more advanced tumor stages and less differentiated HNSCC was observed. Jiang et al (29) found that the promoter of LATS1 was hypermethylated as high as $63.66 \%$ in 88 astrocytomas, indicating that LATS1 may be a useful target for astrocytoma therapy. However, the above studies only reported LATS1 methylation in tumors, did not investigate how LATS1 demethylation affected tumor cells. Therefore, we used $1 \mu \mathrm{M}$ 5-Aza to process $786-\mathrm{O}$ cells for 4 days, and we found LATS1 demethylation could restore its expression and downregulate YAP. It demonstrated that the inactivation of LATS1 was not caused by a genetic alteration, such as mutation, but by a reversible epigenetic mechanism.

We explored the effect of LATS1 overexpression on YAP in 786-O cells. 786-O cells were infected with lentiviral-LATS1 at MOI 60 after $96 \mathrm{~h}$, and the transfection efficiency was $81.69 \%$ by FCM. GFP expression in 786-O cells was high and the exogenous expression of LATS1 strongly downregulated YAP. Its proposed mechanism may be that YAP was phosphorylated at S127 by LATS1 and likely directly interacted by YAP WW domain and LATS1 PPXY motif to activate YAP HXRXXS motif to phosphorylate, which results in YAP binding to 14-3-3 protein and cytoplasmic sequestration (47), YAP might be also phosphorylated by LATS1 kinases at S381, which caused casein kinase 1 (CK1) phosphorylation in succession and recruited ubiquitin factors E3 to degrade YAP in the cytoplasm (48).
The dynamic balance between cell proliferation and apoptosis maintains the normal size of the tissues and organs and the homeostasis of the organisms. However, tumorigenesis which is often related to cell apoptosis is restrained (49). We measured effect of LATS1 overexpression and demethylation on cell apoptosis and proliferation. The results showed the percentage of apoptotic cells in lentiviral-LATS1 groups was higher than other groups and the cell proliferation was inhibited clearly in a time-dependent manner by lentiviral-LATS1. We also found the apoptosis rate of 786-O cells in experiment group was significantly higher than that of control group, and the 786-O cells proliferation was obviously inhibited with $1 \mu \mathrm{M}$ 5-Aza treatment for $96 \mathrm{~h}$, our data for the first time suggested LATS1 overexpression and demethylation obviously induced cell apoptosis and inhibited proliferation in 786-O cells. The mechanism may be through the upregulating pro-apoptosis protein p53 and Bax (50) or enhancing the stability of p53 (51) to induce cell apoptosis and inhibit proliferation.

Regulation of cell cycle is a refined biological process and depends on a series of cell engine molecules which form a complex molecular signal network system, but any one of the molecules or signals which is abnormal will result in disorder of cell cycle regulation and lead to tumorigenesis. LATS1 is a member of the subfamily of protein kinases including Dbf2, Orb6, Cot-1, NDR, and Kpm, which are involved in cell cycle regulation (52), so we investigated the cell cycle by FCM, and found that exogenous expression of LATS1 and demethylation strongly induced cell cycle arrested in G1 stage. Consistent with our notion, Li et al (53) found 3,3'-diindolylmethane (DIM) was able to upregulate expression of LATS1 and induce cell arrest in G1 stage in human gastric cancer cell lines (SNU-1 and SNU-484). Its mechanism is probably that the DIM through MST1/2-LATS1-MOB1 complex promotes an active Hippo signaling pathway and favors YAP phosphorylation. Our studies were different from these research results. Yang et al (50) and Xia et al (54) reported that overexpression of LATS1 caused $\mathrm{G} 2 / \mathrm{M}$ arrest through the inhibition of CDC2 kinase activity in breast cancer cells. Its potential reason may derive from the fact that cell cycle was detected in different tumor cells selected or in different microenvironments of tumor cells.

In conclusion, our research strongly indicates that LATS1 is a TSG in RCC and associated with tumor clinical stage and pathological grade. We are the first to report on that LATS1 is silenced at least in part through promoter hypermethylation in RCC cells. Demethylation of LATS1 promoter by 5-Aza was able to restore the expression by itself, to downregulate YAP, inhibit cell proliferation, induce cell apoptosis and cell cycle arrest in G1 stage. Moreover, overexpression of LATS1 by lentivirus mediation was also able to downregulate YAP, inhibit cell proliferation, induce cell apoptosis and cell cycle arrest in G1 stage. Thus, it would be worth further investigating the possible use of LATS1 methylation as a target for future molecular therapy and diagnosis.

\section{Acknowledgements}

This study was supported by the National Natural Science Foundation of P.R. China (30972999) and Science and Project of Chongqing Municipal Health Bureau (2013-2-082). 


\section{References}

1. Junker K, Ficarra V, Kwon ED, Leibovich BC, Thompson RH and Oosterwijk E: Potential role of genetic markers in the management of kidney cancer. Eur Urol 63: 333-340, 2013.

2. Siegel R, Ward E, Brawley O and Jemal A: Cancer statistics, 2011: the impact of eliminating socioeconomic and racial disparities on premature cancer deaths. CA Cancer J Clin 61 : 212-236, 2011.

3. Linehan WM and Rathmell WK: Kidney cancer. Urol Oncol 30: 948-951, 2012.

4. Motzer RJ, Hutson TE, Tomczak P, et al: Overall survival and updated results for sunitinib compared with interferon alfa in patients with metastatic renal cell carcinoma. J Clin Oncol 27: 3584-3590, 2009.

5. Escudier B, Eisen T, Stadler WM, et al: Sorafenib in advanced clear-cell renal-cell carcinoma. New Engl J Med 356: 125-134, 2007.

6. Cancer Genome Atlas Research Network: Comprehensive molecular characterization of clear cell renal cell carcinoma. Nature 499: 43-49, 2013.

7. Ricketts CJ, Morris MR, Gentle D, et al: Genome-wide CpG island methylation analysis implicates novel genes in the pathogenesis of renal cell carcinoma. Epigenetics 7: 278-290, 2012.

8. Watt F and Molloy PL: Cytosine methylation prevents binding to DNA of a HeLa cell transcription factor required for optimal expression of the adenovirus major late promote. Genes Dev 2: 1136-1143, 1988.

9. Zhu WG, Srinivasan K, Dai Z, et al: Methylator of adjacent $\mathrm{CpG}$ sites affects Sp1/Sp3 binding and activity in the p21(Cip1) promoter. Mol Cell Biol 23: 4056-4065, 2003.

10. Morris MR, Ricketts C, Gentle D, et al: Identification of candidate tumour suppressor genes frequently methylated in renal cell carcinoma. Oncogene 29: 2104-2117, 2010.

11. Reu FJ, Leaman DW, Maitra RR, et al: Expression of RASSF1A, an epigenetically silenced tumor suppressor, overcomes resistance to apoptosis induction by interferons. Cancer Res 66: 2785-2793, 2006.

12. Zhang Q, Ying J, Zhang K, et al: Aberrant methylation of the 8 p22 tumor suppressor gene $D L C 1$ in renal cell carcinoma Cancer Lett 249: 220-226, 2007.

13. Kondratov AG, Stoliar LA, Kvasha SM, et al: Methylation pattern of the putative tumor-suppressor gene $L R R C 3 B$ promoter in clear cell renal cell carcinomas. Mol Med Rep 5: 509-512, 2012.

14. Justice RW, Zilian O, Woods DF, Noll M and Bryant PJ: The Drosophila tumor suppressor gene warts encodes a homolog of human myotonic dystrophy kinase and is required for the control of cell shape and proliferation. Genes Dev 9: 534-546, 1995.

15. Ji T, Liu D, Shao W, Yang W, Wu H and Bian X: Decreased expression of LATS1 is correlated with the progression and prognosis of glioma. J Exp Clin Cancer Res 31: 67, 2012.

16. Zhao B, Tumaneng K and Guan KL: The Hippo pathway in organ size control, tissue regeneration and stem cell self-renewal. Nat Cell Biol 13: 877-883, 2011.

17. Yu T, Bachman J and Lai ZC: Evidence for a tumor suppressor role for the large tumor suppressor genes LATS1 and LATS2 in human cancer. Genetics 195: 1193-1196, 2013.

18. Visser-Grieve S, Zhou Z, She YM, Huang H, Cyr TD, Xu T and Yang X: LATS1 tumor suppressor is a novel actin-binding protein and negative regulator of actin polymerization. Cell Res 21: 1513-1516, 2011.

19. Yabuta N, Mukai S, Okamoto A, et al: N-terminal truncation of Lats1 causes abnormal cell growth control and chromosomal instability. J Cell Sci 126: 508-520, 2013.

20. Visser S and Yang X: Identification of LATS transcriptional targets in HeLa cells using whole human genome oligonucleotide microarray. Gene 449: 22-29, 2010.

21. Zhou GX, Li XY, Zhang Q, et al: Effects of the hippo signaling pathway in human gastric cancer. Asian Pac J Cancer Prev 14: 5199-5205, 2013.

22. Nishio M, Hamada K, Kawahara K, et al: Cancer susceptibility and embryonic lethality in Mobla/1b double-mutant mice. J Clin Invest 122: 4505-4518, 2012.

23. Zhao B, Li L, Wang L, Wang CY, Yu J and Guan KL: Cell detachment activates the Hippo pathway via cytoskeleton reorganization to induce anoikis. Genes Dev 26: 54-68, 2012.

24. St John MA, Tao W, Fei X, et al: Mice deficient of Lats1 develop soft-tissue sarcomas, ovarian tumours and pituitary dysfunction. Nat Genet 21: 182-186, 1999.
25. Yu FX and Guan KL: The Hippo pathway: regulators and regulations. Genes Dev 27: 355-371, 2013.

26. Wierzbicki PM, Adrych K, Kartanowicz D, et al: Underexpression of LATS1 TSG in colorectal cancer is associated with promoter hypermethylation. World J Gastroenterol 19: 4363-4373, 2013.

27. Steinmann K, Sandner A, Schagdarsurengin U and Dammann RH: Frequent promoter hypermethylation of tumor-related genes in head and neck squamous cell carcinoma. Oncol Rep 22: $1519-1526,2009$

28. Seidel C, Schagdarsurengin U, Blümke K, et al: Frequent hypermethylation of MST1 and MST2 in soft tissue sarcoma. Mol Carcinog 46: 865-871, 2007.

29. Jiang Z, Li X, Hu J, Zhou W, Jiang Y, Li G and Lu D: Promoter hypermethylation-mediated down-regulation of LATS1 and LATS2 in human astrocytoma. Neurosci Res 56: 450-458, 2006.

30. Takahashi Y, Miyoshi Y, Takahata C, Irahara N, Taguchi T, Tamaki Y and Noguchi S: Down-regulation of LATS1 and LATS2 mRNA expression by promoter hypermethylation and its association with biologically aggressive phenotype in human breast cancers. Clin Cancer Res 11: 1380-1385, 2005.

31. Tao Q, Huang H, Geiman TM, Lim CY, Fu L, Qiu GH and Robertson KD: Defective de novo methylation of viral and cellular DNA sequences in ICF syndrome cells. Hum Mol Genet 11: 2091-2102, 2002.

32. Hisaoka M, Tanaka A and Hashimoto H: Molecular alterations of h-warts/LATS1 tumor suppressor in human soft tissue sarcoma. Lab Invest 82: 1427-1435, 2002.

33. Nishio M, Otsubo K, Maehama T, Mimori K and Suzuki A: Capturing the mammalian Hippo: elucidating its role in cancer. Cancer Sci 104: 1271-1277, 2013.

34. Bao Y, Hata Y, Ikeda M and Withanage K: Mammalian Hippo pathway: from development to cancer and beyond. J Biochem 149: 361-379, 2011.

35. Schlegelmilch K, Mohseni M, Kirak O, et al: Yap1 acts downstream of $\alpha$-catenin to control epidermal proliferation. Cell 144: 782-795, 2011.

36. Moleirinho S, Chang N, Sims AH, et al: KIBRA exhibits MST-independent functional regulation of the Hippo signaling pathway in mammals. Oncogene 32: 1821-1830, 2013.

37. Angus L, Moleirinho S, Herron L, et al: Willin/FRMD6 expression activates the Hippo signaling pathway kinases in mammals and antagonizes oncogenic YAP. Oncogene 31: 238-250, 2011

38. Badouel $\mathrm{C}$ and McNeill H: SnapShot: the hippo signaling pathway. Cell 145: 484-484, 2011.

39. Visser S and Yang X: LATS tumor suppressor. a new governor of cellular homeostasis. Cell Cycle 9: 3892-3903, 2010.

40. Salah Z, Cohen S, Itzhaki E and Aqeilan RI: NEDD4 E3 ligase inhibits the activity of the Hippo pathway by targeting LATS1 for degradation. Cell Cycle 12: 3817-3823, 2013.

41. Serrano I, McDonald PC, Lock F, Muller WJ and Dedhar S: Inactivation of the Hippo tumour suppressor pathway by integrin-linked kinase. Nat Commun 4: 2976, 2013.

42. Mo JS, Yu FX, Gong R, Brown JH and Guan KL: Regulation of the Hippo-YAP pathway by protease-activated receptors (PARs). Genes Dev 26: 2138-2143, 2012.

43. Yu FX, Zhao B, Panupinthu N, et al: Regulation of the Hippo-YAP pathway by G-protein-coupled receptor signaling. Cell 150: 780-791, 2012.

44. Noviello C, Courjal F and Theillet C: Loss of heterozygosity on the long arm of chromosome 6 in breast cancer: possibly four regions of deletion. Clin Cancer Res 2: 1601-1606, 1996.

45. Lee EY, To H, Shew JY, Bookstein R, Scully P and Lee WH: Inactivation of the retinoblastoma susceptibility gene in human breast cancers. Science 241: 218-221, 1988.

46. Morinaga N, Shitara Y, Yanagita Y, et al: Molecular analysis of the $h$-warts/LATS1 gene in human breast cancer. Int J Oncol 17: $1125-1129,2000$.

47. Hong W and Guan KL: The YAP and TAZ transcription co-activators: key downstream effectors of the mammalian Hippo pathway. Semin Cell Dev Biol 23: 785-793, 2012.

48. Hergovich A: Regulation and functions of mammalian LATS/NDR kinases: looking beyond canonical Hippo signalling. Cell Biosci 3: 32, 2013.

49. Valis K, Prochazka L, Boura E, et al: Hippo/Mst1 stimulates transcription of the proapoptotic mediator NOXA in a FoxO1-dependent manner. Cancer Res 71: 946-954, 2011.

50. Yang X, Li DM, Chen $\mathrm{W}$ and $\mathrm{Xu} \mathrm{T}$ : Human homologue of Drosophila lats, LATS1, negatively regulate growth by inducing $\mathrm{G}(2) / \mathrm{M}$ arrest or apoptosis. Oncogene 20: 6516-6523, 2001. 
51. Matallanas D, Romano D, Al-Mulla F, et al: Mutant K-Ras activation of the proapoptotic MST2 pathway is antagonized by wild-type K-Ras. Mol Cell 44: 893-906, 2011.

52. Hori T, Takaori-Kondo A, Kamikubo Y and Uchiyama T: Molecular cloning of a novel human protein kinase, $\mathrm{kpm}$, that is homologous to warts/lats, a Drosophila tumor suppressor. Oncogene 19: 3101-3109, 2000.
53. Li XJ, Park ES, Park MH and Kim SM: 3,3'-Diindolylmethane suppresses the growth of gastric cancer cells via activation of the Hippo signaling pathway. Oncol Rep 30: 2419-2426, 2013.

54. Xia H, Qi H, Li Y, et al: LATS1 tumor suppressor regulates G2/M transition and apoptosis. Oncogene 21: 1233-1241, 2002. 